\title{
Proceeding
}

Supplementary Issue: Spring Conferences of Sports Science. First International Conference in Iraq on Sport for Peace, 4 April 2019. Baghdad Science Institute, Baghdad, Iraq.

\section{Semi - regular exercises for the rehabilitation of shoulder joint for weightlifting disabled athletes}

\author{
DHAFIR HARB EWAJELA AL-IBRAHEEMI ${ }^{1}$, JAMEEL QASIM MOHAMMED AL-BADRI ${ }^{2}$, AQEEL \\ HAMEED OUDAH AL-OUDAH ${ }^{3}$ \\ ${ }^{1}$ College of Physical Education and Sports Sciences, University of Baghdad, Iraq \\ ${ }^{2}$ Department of Student Activities, Presidency of the University of Baghdad, Iraq \\ International Relations and Cooperation Department, Ministry of Youth and Sports, Iraq
}

\begin{abstract}
The weight lifting athletes get injured while they are demonstrating the strength to lift the weight. It is observed mostly the shoulder joints are prone to affect due to physical imbalance or abnormal movement in weightlifting athletes. This results impair normal functional movement. The rehabilitation centers are providing the semiregular exercises to regain their muscular strength. Muscular endurance is predominant rather than muscular strength. The rehabilitation centers are initially focusing on retain their muscular endurance and then focusing on increasing the muscular strength by practicing the semi-regular exercises. In this research work we suggest the semi-regular exercises to improve 3 different muscle contractions namely isometric contraction, concentric contraction and eccentric contractions of the skeletal muscle. The semi-regular exercises are aiming to improve the joint strength and range of motion in shoulder joint dislocation. This can be provided after two weeks of the shoulder dislocation of the weightlifting athletes with the confirmation from physician. The duration of the semi-regular exercises rehabilitation program is spanned across the period of 6 weeks to regain their muscle power and strength. Keywords: Weightlifting; Muscular strength; Muscular endurance; Semi-regular exercise.

Cite this article as:

Al-lbraheemi, D.H.E., Al-Badri, J.Q.M., \& Al-Oudah, A.H.O. (2019). Semi - regular exercises for the rehabilitation of shoulder joint for weightlifting disabled athletes. Journal of Human Sport and Exercise, 14(4proc), S477-S487. doi:https://doi.org/10.14198/ihse.2019.14.Proc4.06

Corresponding author. Department of Student Activities, Presidency of the University of Baghdad, Iraq.

E-mail: Jameel 79j@yahoo.com

Supplementary Issue: Spring Conferences of Sports Science. First International Conference in Iraq on Sport for Peace, 4 April 2019. Baghdad Science Institute, Baghdad, Iraq.

JOURNAL OF HUMAN SPORT \& EXERCISE ISSN 1988-5202

(c) Faculty of Education. University of Alicante

doi:10.14198/jhse.2019.14.Proc4.06
\end{abstract}




\section{INTRODUCTION}

The dislocation of shoulder joints are frequently seen in the weightlifting athletes while performing the practice with physical imbalance or sometime abnormal movements in weightlifting functional movements. The most common weightlifting injuries are disc herniation, Achilles tendonitis, Superior Labrum Anterior and Posterior tear, Lateral Elbow Tendonitis, Hamstring Pull and Tears, Patellar Tendonitis and Knee pain finally IT Band Syndrome. These injuries might happen when the athletes are not following the basic rules when they are in hurry and when they are doing with lack of attention. The injuries are accidental not intentional. The trainers will be very cautious to prepare the athletes to do sufficient warm-up exercises before practicing the weightlifting practices. The athletes are instructed to concentrate on their weightlifting practice with proper attention and concentration. The injuries can occur when the athletes are derailed from their concentration and balance (Baudisch \& Naumann, 2018).

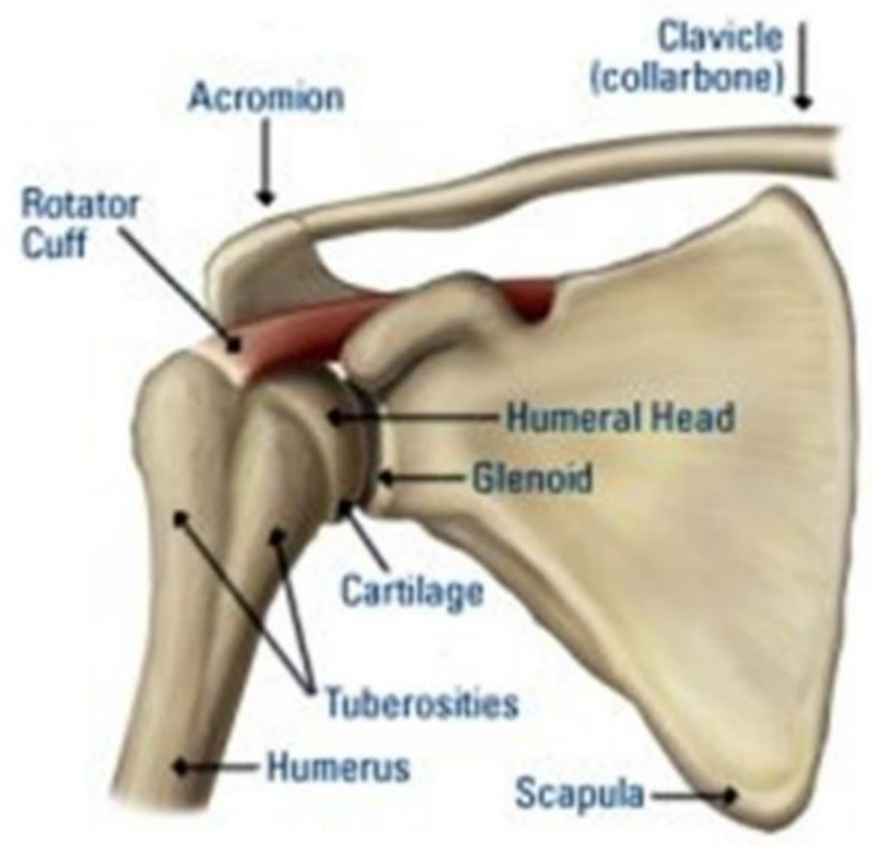

Figure 1. Physiology of shoulder

It is very important to understand the physiology of shoulder joints how it is organized. When the ball of upper arm bone humerus is forced fully out of its normal position on the shoulder socket glenoid labrum. It will happen when the athletes perform the exercise in a vulnerable position at a popped out speed. Sometimes it may occur at repetitive overstretching of the shoulder joint. The joint dislocation can be happened in two types (Velloso et al,2013). These are anterior dislocation and posterior dislocation. When the relocation is done by the physiotherapists or medical practitioners without any surgical rehabilitation. When the surgical operations are done the rehabilitation period will be increased to 3 to 6 months. It is done without surgical operation the resting period will be two weeks (Gaballah et al,2017). 


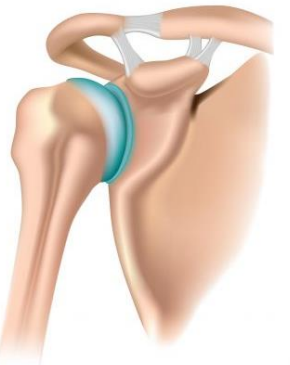

Normal anatomy

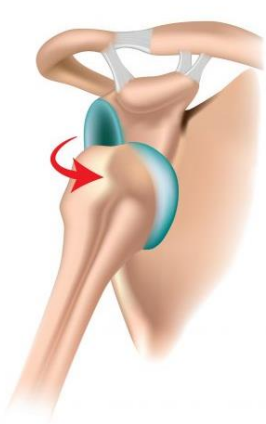

Anterior dislocation

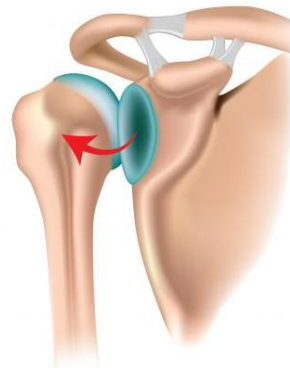

Posterior dislocation

Figure 1bis. Shoulder Dislocation

When the dislocation of shoulder occurs the athletes experiences extreme pain at shoulder. They will experience the inability to move their arm until the dislocated part is relocated back into the socket. The resident physicians or experienced trainers will adjust the shoulder and make the dislocated part to its original position. Though the relocation is done for the ball of the humerus, the athletes experience remarkable pain in their shoulders. They have to take rest for two weeks without making any movement of the affected hand. This can be done by the physicians with the help of tima adjustable orthopaedic shoulder support neoprene. The resting position will be continued for two weeks and the body should regain the muscle power with the help of semi-regular exercises in the rehabilitation centre prescribed for weightlifting athletes. This can be same for without surgical operational correction or with surgical operational correction for relation of the (Andersen et al, 2016).

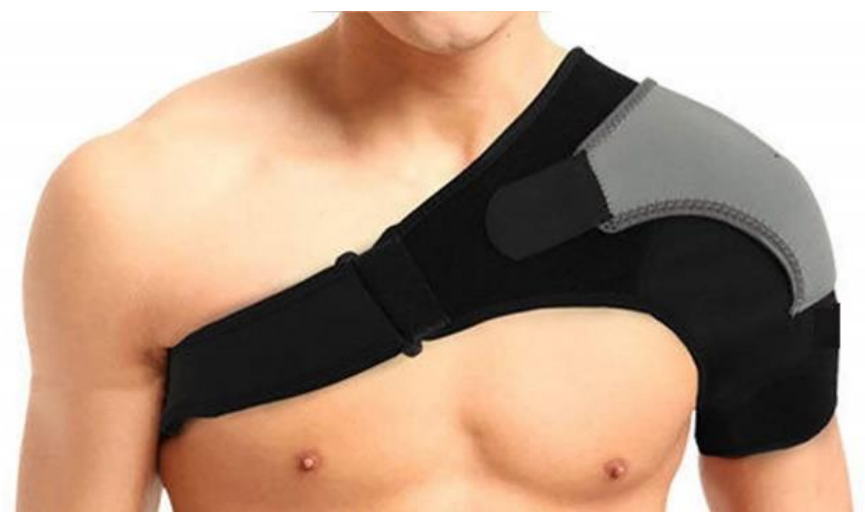

Figure 2. Tima - shoulder support neoprene

In the proposed research paper we propose six week semi-regular exercises in the rehabilitation centre with scientifically proven methodology.

\section{METHODOLOGY}

We propose semi regular exercises for the rehabilitation of shoulder joint dislocated athletes for period of 6 weeks (Andersen et al, 2016). This is a proven scientific methodology to regain the power of muscle. Once 
the head of humerus is properly sit in the position with the Glenold Fossa. The Deltoid Muscle which is formulated around the subdeltoid bursa and articular capsule will be affected and needs to get revived. The power of long head of triceps muscle and teres major muscles are needed to get strengthen to perform weightlifting. In this process we suggest establishing the muscular endurance with the help of easy semiregular exercise at first. Then we concentrate on muscular strength building (Badr \& Gaballah,2015).

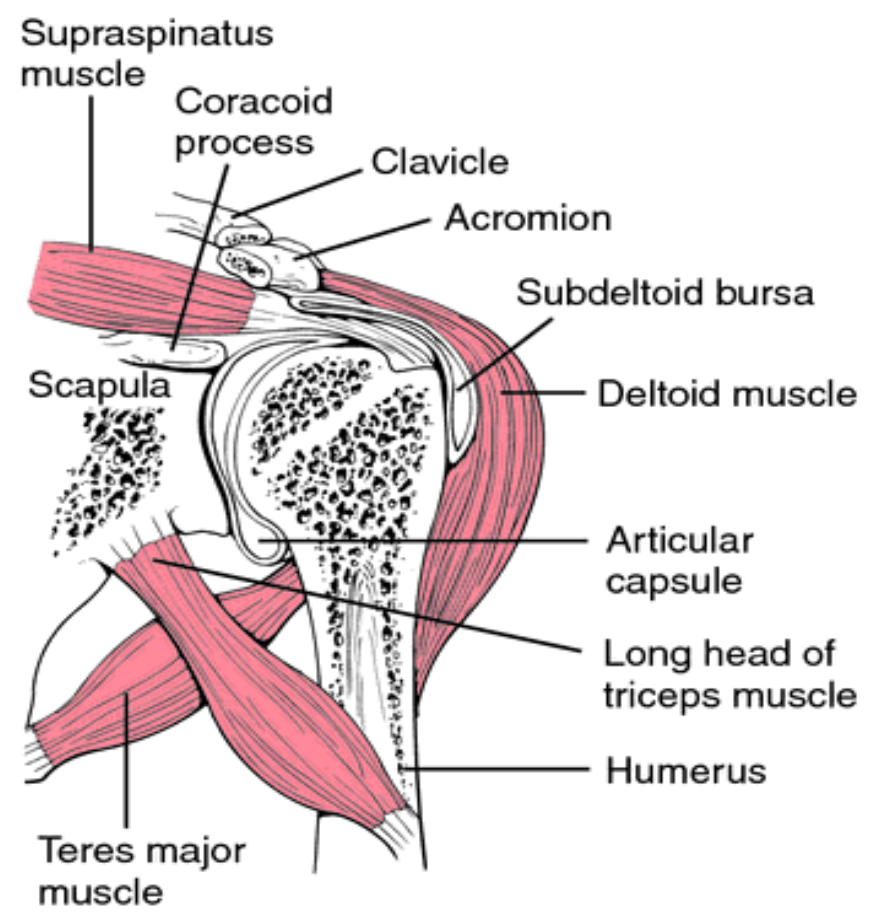

Figure 3. Physiology of Shoulder muscle architecture

The shoulder dislocation can affect three parts of the joint of exoskeleton. Joint 1 Joint 2 and Joint 3 as shown in the below figure. The dislocation can be relocated but the strength of the muscle needs to be restored within a stipulated time period. For this we suggest 6 weeks of time (Gale,2007).

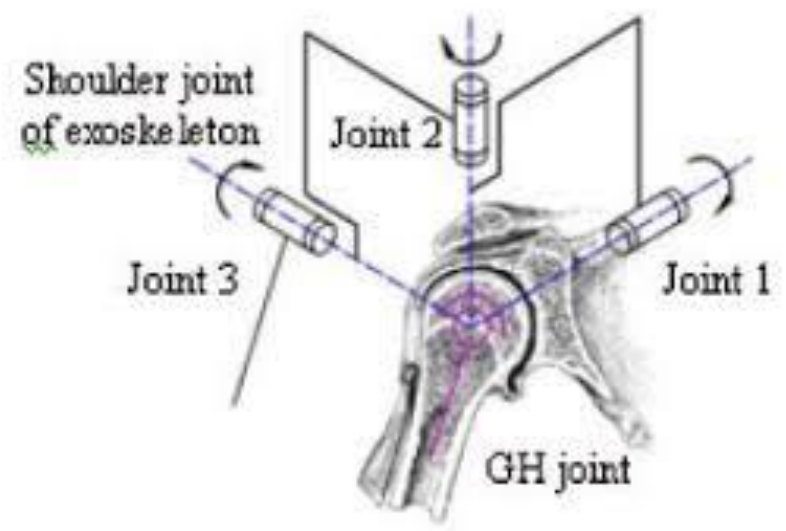

In this process we suggest the severity of the pain in shoulder joint. The physiotherapist can check the severity and suggest semi regular exercises for the rehabilitation (Andersen et al, 2016). 


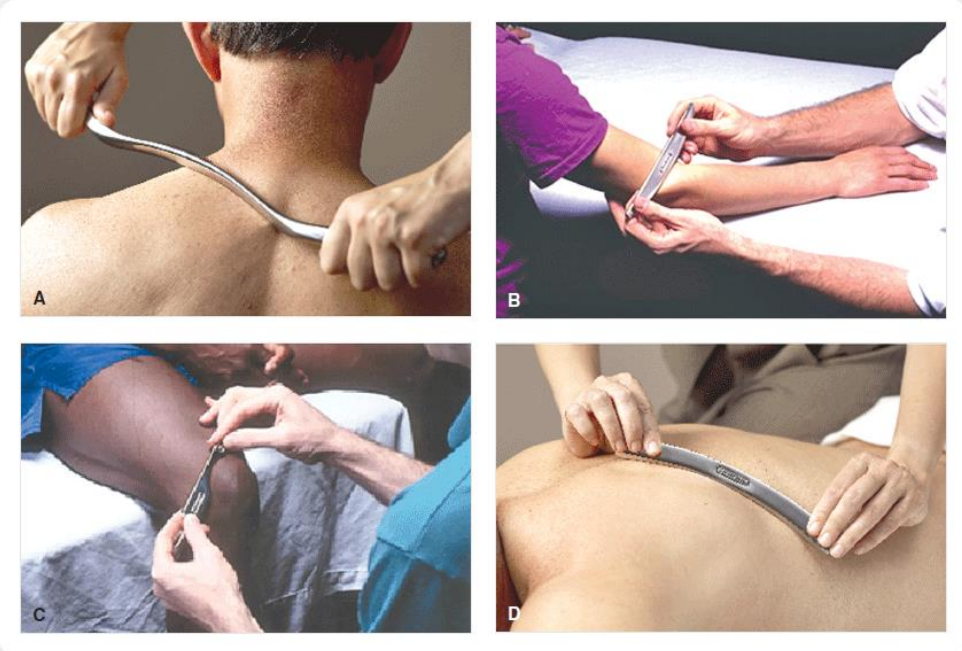

The rehabilitation is divided into three stages. In the first sage the muscle endurance is given importance. In the second stage the muscle strength is focused. The third stage is orchestrated to improve the power of the muscle. in the muscle strength development we focus on three different muscle contractions namely isometric contraction, concentric contraction and eccentric contractions of the skeletal muscle (Nagata et al, 2015).

Stage one is purely concentrating to retain the endurance of the muscle with easy exercises. This exercise will make the hand free movement and retain the muscle endurance. This simple exercise can be practiced for a couple of days to regain the free hand movement and reduced shoulder pain (Liu et al, 2014).
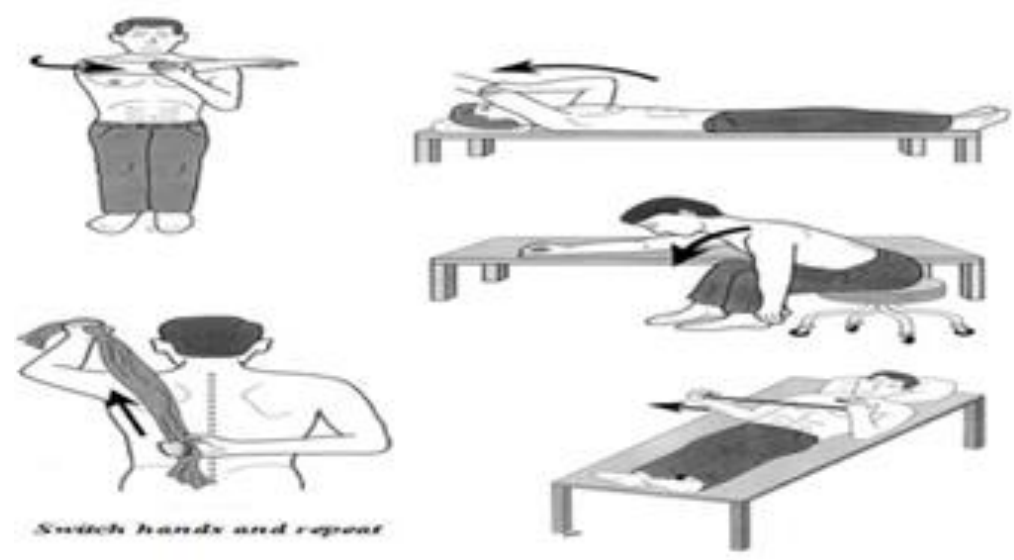

Figure 4. Easy exercise for muscle endurance and free hand movement

In the building of muscle power we suggest the Isometric contraction with easy exercise with dumbles lifting. Dumbles lifting with smaller amount of weight to greater amount of weight can increase the power of the muscle and strengthen the hand. Biceps is the best example to increase the strength of the muscle in the sage two (Mascarin et al, 2016). 


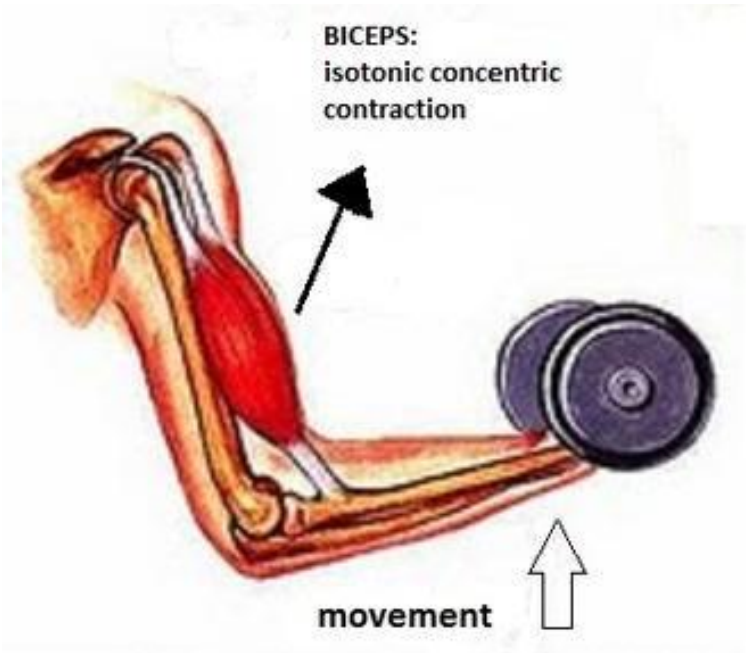

Figure 5. Biceps for isometric contraction

In the second stage the concentric contraction and eccentric contraction of the skeletal muscle is focused. This will bring back the muscle strength. This may be practiced for a period of two weeks and added with third stage of exercises. The third stage of exercise will make the power of the muscle improvement (Kenneth, 2017).

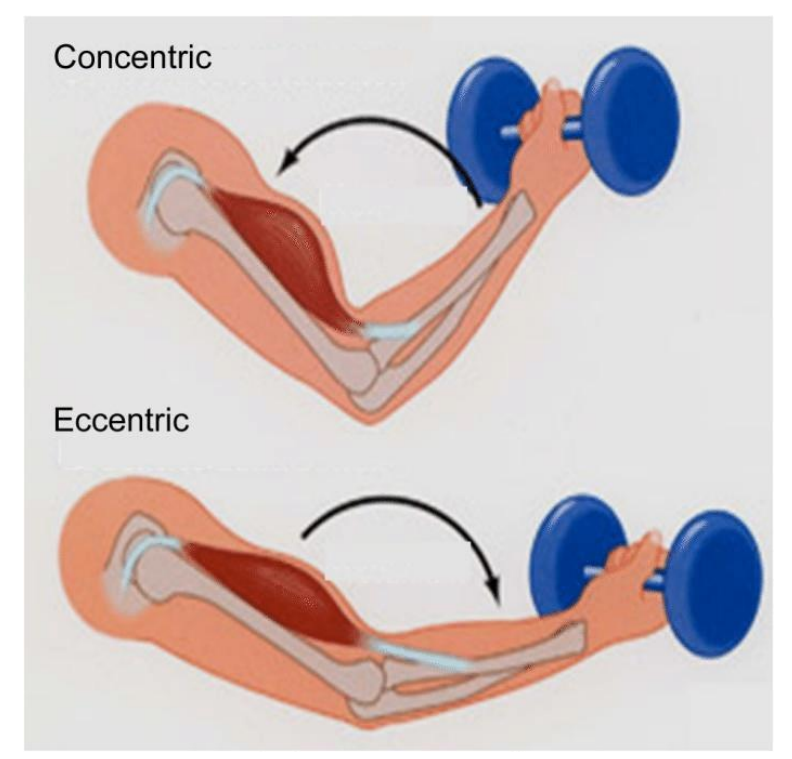

Figure 6. Exercise focusing concentric and eccentric

The third stage will be continued up to $6^{\text {th }}$ week to get back the original capacity of the shoulder to sustain the weightlifting with great ease. The second stage biceps will be continued up to sixth week to make the muscle improvisation (Stephen, 2017). 

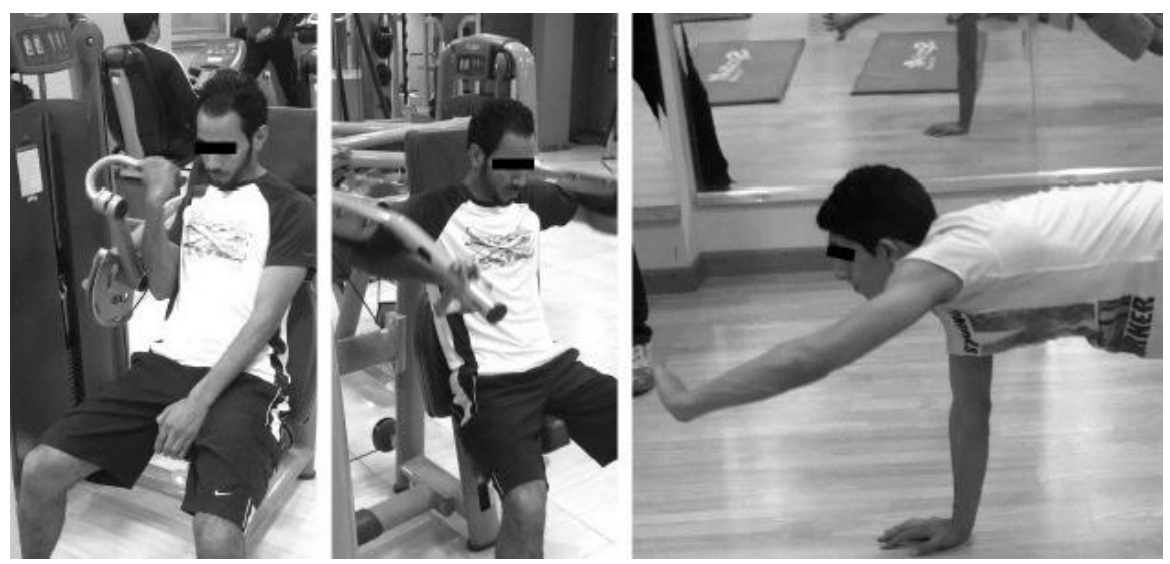

Figure 7. Semi regular exercises for regain the power of shoulder

Semi-regular exercises are mandatory for improving the shoulder joints for weightlifting athletes. The following simple and easy exercise also needs to be practiced by the weightlifting athletes in the rehabilitation centre to get the power of shoulder strength (Proske \& Gandevia, 2012).

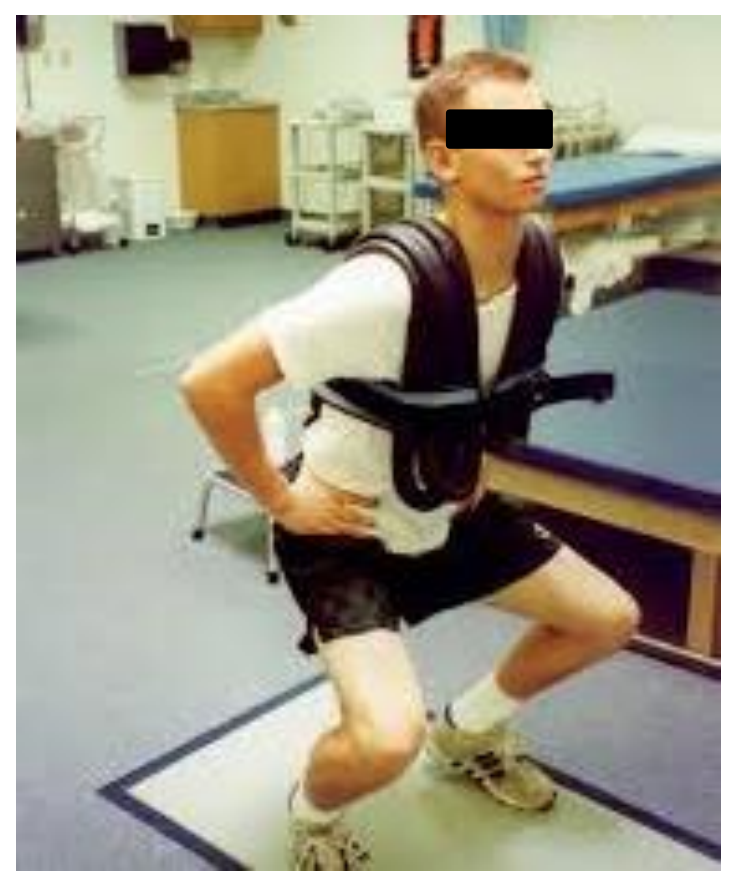

Figure 8. Shoulder strength improvement for weightlifting

Stretching exercises can help the shoulder reviving and strength building. The semi-regular exercises with stretching the hands and pushing the shoulders on the balloons can help to improve the strength of the muscles. The combination of exercise should be done for next duration of the tenure (Soleimani et al,2014). 

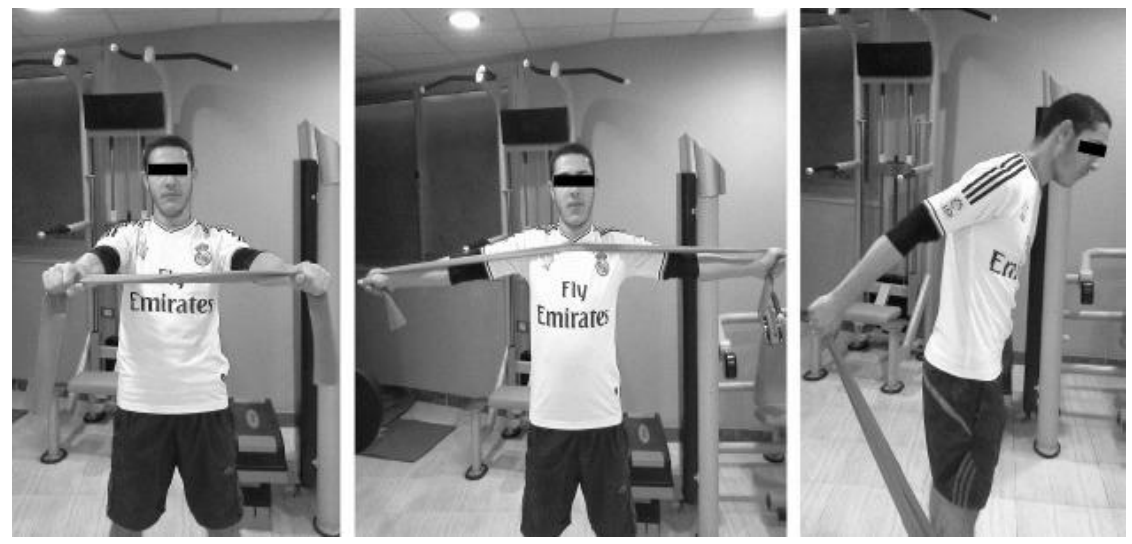

Figure 9. Stretching exercises for shoulder strength

The methodology to improve the shoulder muscle strength is proven with scientific process of physiotherapy. The following graph can reveal the systematic improvement of the muscle strength to perform the weightlifting after 6 weeks (Andersen et al, 2016).

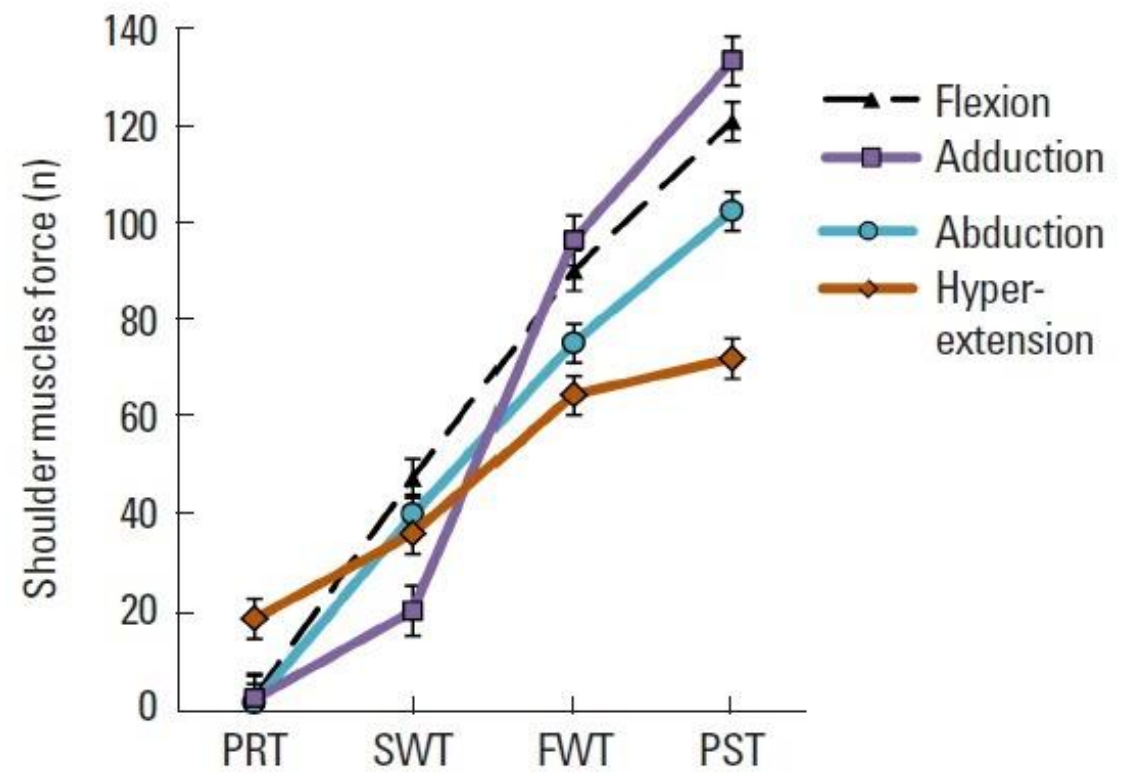

Figure 10. Shoulder muscles force improvement

The results have been extracted from 10 weightlifting athletes with proper exercise to streamline the strength in three stages. PRT indicates the Pretest and SWT is indicating the Second Week Test and FWT indicating the Fourth Week Test and finally PST is taken for Posttest results for the athletes who have experienced the shoulder dislocation (Gest, 2017). 


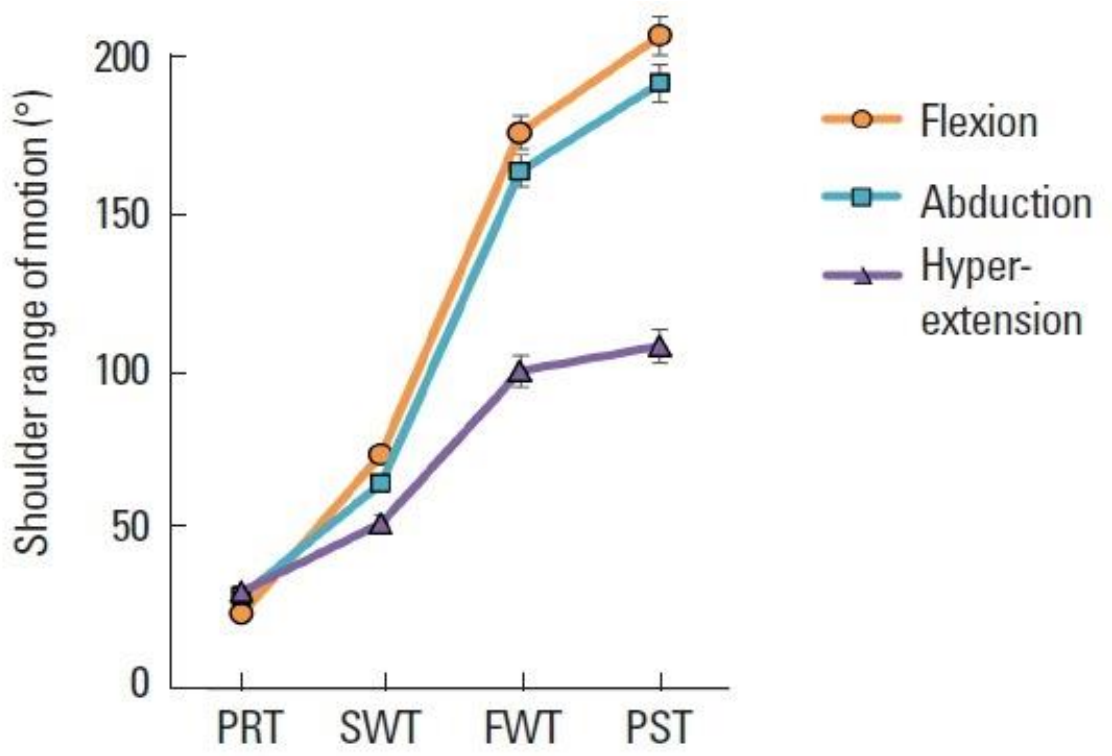

Figure 1. Shoulder motion improvement

The above picture demonstrates the shoulder range motion. When the shoulder is injured with shoulder joint dislocation the shoulder motion is nullified for two weeks. Then in the rehabilitation period the shoulder motion is improvised gradually with scientific methodology. The results have revealed the shoulder motion has remarkably increased and bring back the original state of the athletes to perform the weightlifting after 6 weeks (Andersen et al, 2016).

\section{THE SOLUTION}

The solution can be obtained from three stages. In the first week of the exercise protocol the muscle endurance has been achieved. This is in the first stage.

In the second stage the muscle strength is concentrated and required semi regular exercise protocol is provided to increase the strength of the shoulder muscle from 0 level to required level.

In the third stage the increased exercise protocol is suggested to increase the power of the muscles. The third stage has given enough and proven solution for the problems faced by the shoulder dislocation suffering weightlifting athletes from fourth week to sixth week. The obtained results have demonstrated the power of muscles in relation with flexion, adduction, abduction and Hyper extension (Anterior, 2016).

In this solution we suggested 6 week exercise protocol to improve the shoulder motion and should power. This has been proved with scientific methodology of exercises after analysing the shoulder muscle anatomy and physiology. The suggested exercises spanned across 6 weeks are going to strengthen the glenohumeral joint and increase the grip on the socket of the scapula. The circular movement of the arm is improved in the suggested semi-regular exercises. The suggested exercises are aiming at improving the labrum which can be covered the glenohumeral joint and exist between the ball and socket of the shoulder joint. The ball and socket construction is strengthened in the second week to sixth week exercises. The results of the exercises are increase the strength of the clavicle and acromion in the acromioclavicular joint. The function of the shoulder joint will be regulated in the first half of the week. From the second week onwards the strength of 
the associated muscles covered the shoulder joints and bony projection off the scapula are increased from the initial level to required level to participate the regular practice of weightlifting (shoulder instability, 2018).

\section{CONCLUSION}

The predominant problem with weightlifting athletes is shoulder joint injury. This is experienced while they are practicing the weigh lift with wrong angle or less attention or attentiveness. The shoulder joints are very seriously considered and affect the athletes with great pain. In this research work we proposed a six week exercise protocol for reviving the weightlifting athletes to enforce normal practice of their day to day weightlifting (Dislocated Shoulder, 2018). We suggested the rehabilitation centres where the semi-regular exercises to regain their muscular endurance as well as muscular strength. In our suggested due course we initially focused on retain their muscular endurance. The next due course which is starting in the second week is focusing on muscular strength by practicing the semi-regular exercises. In this research work we suggested the 3 different muscle contractions in three different stages (Ghazanfarpour et al,2013). In this research work we demonstrated the results with solutions to regain the muscular strength in all aspects of required. The duration of the semi-regular exercises rehabilitation program is spanned across the period of 6 weeks to regain their muscle power and strength and to reconstruct the glenoid and coracobrachialis attachments.

\section{REFERENCES}

Proske, U., \& Gandevia, S. C. (2012). The proprioceptive senses: their roles in signaling body shape, body position and movement, and muscle force. Physiological reviews, 92(4), 1651-1697. https://doi.org/10.1152/physrev.00048.2011

Patrick Baudisch., \& Felix Naumann. (2018). interactive systems based on electrical muscle stimulation published by Institutional Repository of the University of Potsdam.

Eduardo Velloso, Andreas Bulling, Hans Gellersen, Wallace Ugulino,Hugo Fuks. ( 2013). Qualitative activity recognition of weight lifting exercises published in $\mathrm{AH}$ '13 Proceedings of the 4th Augmented Human International Conference Stuttgart, Germany - March 07 - 08, 2013 ACM New York, NY, USA @2013 URN urn:nbn:de:kobv:517-opus4-421165. https://doi.org/10.1145/2459236.2459256

Gaballah, A., Zeyada, M., Elgeidi, A., \& Bressel, E. (2017). Six-week physical rehabilitation protocol for anterior shoulder dislocation in athletes. Journal of exercise rehabilitation, 13(3), 353. https://doi.org/10.12965/jer.1734976.488

Thomson Gale. (2007). Shoulder Anatomy And Physiology published by World of Sports Science Copyright 2007 Thomson Gale.

Andersen, L. L., Vinstrup, J., Jakobsen, M. D., \& Sundstrup, E. (2017). Validity and reliability of elastic resistance bands for measuring shoulder muscle strength. Scandinavian journal of medicine \& science in sports, 27(8), 887-894. https://doi.org/10.1111/sms.12695

Badr, M. A., \& Gaballah, A. M. (2015). Common injuries among male field hockey players according to playing positions. Journal of Applied Sports Science, 5(1).

Liu, A., Xue, X., Chen, Y., Bi, F., \& Yan, S. (2014). The external rotation immobilisation does not reduce recurrence rates or improve quality of life after primary anterior shoulder dislocation: a systematic review and meta-analysis. Injury, 45(12), 1842-1847. https://doi.org/10.1016/j.injury.2014.06.005

Mascarin NC, de Lira CA, Vancini RL, de Castro Pochini A, da Silva AC, Dos Santos Andrade M. (2016). Strength training using elastic band improves muscle power and throwing performance in young female handball players. Published in Journal of Sports Rehabilitation.1-25. https://doi.org/10.1123/jsr.2015-0153 
Nagata, H., Thomas, W. J., \& Woods, D. A. (2016). The management of secondary frozen shoulder after anterior shoulder dislocation-The results of manipulation under anaesthesia and injection. Journal of orthopaedics, 13(2), 100-105. https://doi.org/10.1016/i.jor.2015.01.030

Kenneth Leung. (2017). 7 Most Common Weight Lifting Injuries (\& How To Prevent Them) published in builtlean.com.

Stephen Kishner, MD, MHA. (2017). Shoulder Joint Anatomy published the Updated version.

Thomas R Gest,. (2017). shoulder pain non-operative rehabilitation guidelines published in Sports Medicine. Connecticut Children's Medical Center.

Hossein Ghazanfarpour, Mohsen Pourkhosravani, S. Elham Mousavi. (2013). Geomorphic systems affecting the Kerman. UCT Journal of Social Sciences and Humanities Research. Issue 4.

Mahshid, Soleimani ., Hamidreza, Nezhadali lafmejani. , Zahra, Mohammad Beigi. (2014). Effective indicators in Bank customer satisfaction. UCT Journal of Social Sciences and Humanities Research. Issue 1. 